\title{
Clinicopathological Study of Nonsquamous Cell Malignancies of the Head and Neck
}

\author{
Vipin Ram Ekhar, Devendra M Mahore, Gauri M Kapre, Bhagyashree D Bokare, Surendra H Gawarle
}

\begin{abstract}
There is a tendency among the head and neck surgeons to classify head and neck tumors as squamous and nonsquamous. The latter is a miscellaneous collection of less common, but certainly no less significant neoplasms. The rarity of these tumors is evident from the limited data available on clinical characteristics and outcomes. This study is thus an attempt to meet the need and fill the void by providing practical information regarding different pathological subtypes, their treatment and prognosis. In our study, we documented a total of 48 cases of nonsquamous cell cancers of the head and neck region including all sites like nose and paranasal sinuses, oral cavity and oropharynx, neck and larynx and documented important clinical, pathological and treatment features of these.
\end{abstract}

Keywords: Nonsquamous malignancies, Head and neck, Pathological types, Management modalities.

How to cite this article: Ekhar VR, Mahore DM, Kapre GM, Bokare BD, Gawarle SH. Clinicopathological Study of Nonsquamous Cell Malignancies of the Head and Neck. Int $\mathrm{J}$ Head and Neck Surg 2013;4(2):70-73.

Source of support: Nil

Conflict of interest: None

\section{INTRODUCTION}

Head and neck malignancies are among the most devastating disease processes we encounter, and treatment of these head and neck cancers is complex. Accepted best management can vary significantly based upon seemingly subtle differences in tumor location and histopathology. Nonsquamous malignancies of the head and neck are a miscellaneous collection of less common, but certainly no less significant neoplasms. The rarity of these tumors is evident from the limited data available on clinical characteristics and outcomes. In addition to the sporadic occurrences, the clumping of small number of patients of nonsquamous cell cancers with squamous cell cancers in most reported series of head and neck malignancies and the absence of prospective treatment protocol are all responsible for the paucity of published literature for these cancers. There is therefore a need for relevant data on clinical characteristics, treatment and outcome for nonsquamous cell cancers of the head and neck, which will be useful in counseling newly diagnosed patients, with regards to management and prognosis.

\section{MATERIALS AND METHODS}

The hospital-based prospective clinical study was undertaken in the Department of Otolaryngology,
Government Medical College, Nagpur, from June 2006 to November 2008 with the following aims:

1. To create a comprehensive database about the nonsquamous cell malignancies of the head and neck.

2. To know the various histopathological subtypes.

3. To study which among these are the more common ones.

4. To study tumor behavior and response to treatment of these subtypes.

5. To study the morbidity and mortality due to these tumors. All patients who were proved to have nonsquamous cell cancers of the head and neck region, either by cytology or by histopathology, were included in the study. All squamous cell tumors and their variants, all benign tumors of the head and neck and all tumors arising from the major salivary glands and thyroid were excluded from the study group.

After a detailed head and neck examination, relevant pathological investigations like fine needle aspiration cytology (FNAC), incisional biopsy or excisional biopsy, were done and the pathological subtype of the tumor was noted. Appropriate treatment modality like surgery, radiotherapy and chemotherapy, individually or in combination, were instituted for each case. Cases were followed up at 1, 3, 6 months and 1 year following treatment. Any complications of treatment received or morbidity and mortality were noted during follow-up. Response to treatment was judged as 'complete response' (no residual tumor at completion of treatment), 'residual disease' (regression in tumor size but presence of residue) and 'recurrence' of tumor.

\section{OBSERVATIONS AND RESULTS}

During our study period of 2.5 years we documented a total of 48 cases which included 31 men and 17 women (M:F ratio, 1.82:1). The age incidence of all the cases showed that majority of the patients belonged to the age group of 50 to 60 years (10 patients) followed by the age group of 60 to 70 years (eight patients) and 40 to 50 years (eight patients). Notably there were seven patients in the 10 to 20 years age group. The youngest patient was 6 years old who was a case of non-Hodgkins' lymphoma of the maxilla and the oldest patient was 84 years old, a case of esthesioneuroblastoma.

The site in the head and neck which was involved by the tumor and the specific subsites were noted (Table 1). It 
Clinicopathological Study of Nonsquamous Cell Malignancies of the Head and Neck

\begin{tabular}{clcc}
\hline \multicolumn{3}{c}{ Table 1: Site of tumors } \\
\hline Sr. no. Site & $\begin{array}{c}\text { No. of } \\
\text { cases }\end{array}$ & Percentage \\
\hline & & \\
\hline 1. & Nose and paranasal sinuses & 30 & 62.5 \\
& a. Maxillary sinus & 22 & 45.83 \\
& b. Ethmoidal sinus & 2 & 4.17 \\
& c. Nasal cavity & 5 & 10.42 \\
d. External nose & 1 & 2.08 \\
2. Oral cavity and oropharynx & 11 & 22.92 \\
& a. Palate & 3 & 6.25 \\
& b. Tonsil & 3 & 6.25 \\
& c. Mandible & 3 & 6.25 \\
& d. Buccal mucosa & 1 & 2.08 \\
e. Tongue & 1 & 2.08 \\
3. Neck & 5 & 10.42 \\
4. Larynx & 1 & 2.08 \\
5. Nasopharynx & 1 & 2.08 \\
\hline
\end{tabular}

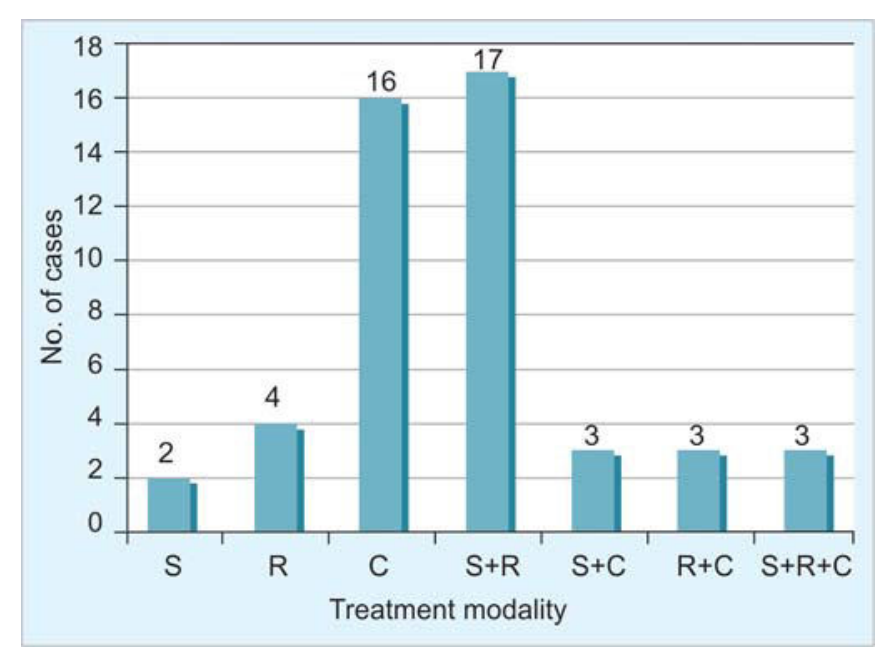

Fig. 1: Treatment employed

was found that most of the tumors ( 30 cases) originated in the nose and paranasal sinuses (62.5\%). Maximum numbers of malignancies were located in the maxillary sinus (45.83\%). The next most commonly involved site was the oral cavity and oropharynx with three cases each of the tonsil, palate and mandible. We came across one case each of small cell tumors of the larynx (glottis) and Hodgkins' lymphoma of the nasopharynx with neck secondaries. There was no case involving the ear.

A wide range of pathological diagnoses were encountered (Table 2). The commonest of these was lymphoma (25\%) of which seven were non-Hodgkins' lymphoma and five were Hodgkins' lymphoma. The next most common diagnosis was adenoid cystic carcinoma (20.83\%). Other types included adenocarcinoma (12.5\%), mucosal melanoma and esthesioneuroblastoma ( $10.42 \%$ each).

Of the 30 cases of the nose and paranasal sinuses tumors, adenoid cystic carcinoma was the most commonly encountered malignancy (33.33\%) and it was found maximally in the maxillary sinus ( 9 out of 10 cases). The second common pathology was adenocarcinoma, five cases,

\begin{tabular}{clcc}
\multicolumn{4}{c}{ Table 2: Pathology of tumors } \\
\hline Sr. no. & Pathological subtype & No. of cases & Percentage \\
\hline 1. & Lymphoma & 12 & 25.00 \\
2. & Adenoid cystic & 10 & 20.83 \\
& carcinoma & & \\
3. & Adenocarcinoma & 6 & 12.50 \\
4. & Mucosal melanoma & 5 & 10.42 \\
5. & Esthesioneuroblastoma & 5 & 10.42 \\
6. & Rhabdomyosarcoma & 2 & 4.17 \\
7. & Ewing's Sarcoma & 2 & 4.17 \\
8. & Basal cell carcinoma & 1 & 2.08 \\
9. & Malignant schwannoma & 1 & 2.08 \\
10. & Small-cell carcinoma & 1 & 2.08 \\
11. & Hemangiopericytoma & 1 & 2.08 \\
12. & Acinic cell carcinoma & 1 & 2.08 \\
13. & Osteosarcoma & 1 & 2.08 \\
\hline
\end{tabular}

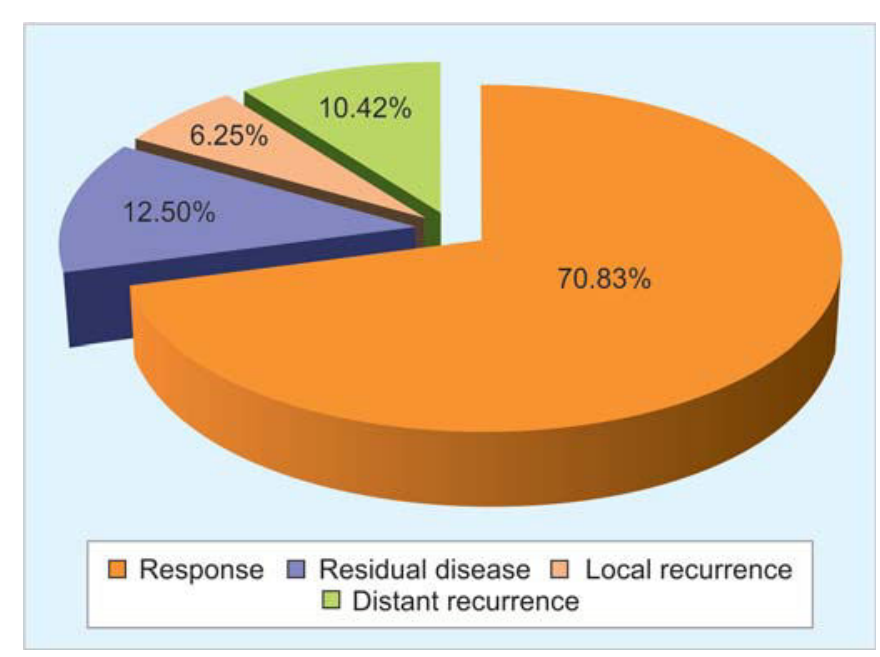

Fig. 2: Response to treatment

all of which were found in the maxilla. Interestingly, in our study period we did not come across a case of adenocarcinoma of the ethmoid sinus. Esthesioneuroblastomas (five cases) were all found in the nasal cavity whereas malignant melanomas were found in the maxillary sinus (three cases) and ethmoid sinus (one case).

Depending upon the site and type of tumor, single modality or multimodality treatment was given as indicated in each particular case (Fig. 1). Some cases were initially given a single modality treatment and if the response was not favorable a second treatment option was initiated as appropriate. It was found that the most frequently employed therapy was surgery + radiotherapy which was implemented in 17 cases (35.42\%) followed by chemotherapy alone which was implemented in 16 cases $(33.33 \%)$ which was due to the high incidence of lymphomas which are exquisitely chemosensitive. Surgery alone was done in only two cases as in both these cases patients were not willing for postoperative $\mathrm{CT} / \mathrm{RT}$.

The response to treatment (Fig. 2) was judged during follow-up at 1 month, 6 months and 1 year. Most of the 
patient showed excellent response and complete resolution of disease at follow-up. The response was graded as follows:

1. Resolution of disease at the end of treatment.

2. Residual disease after completion of treatment.

3. Recurrence of the disease after completion of treatment, either at the locoregional level or at a distant site.

As regards to the behavior of major individual subtypes of the tumors, it was seen that nine out of the 12 cases of lymphomas showed response to treatment while three had recurrences at distant sites. Adenoid cystic carcinomas which the next most commonly encountered tumor also showed good response with eight out of 10 patients showing complete resolution of the tumor at the completion of treatment. Cases of small-cell tumor, acinic cell carcinoma, osteosarcoma and Ewings' sarcoma showed complete response to treatment while esthesioneuroblastomas were locally advanced at presentation and three of five cases showed residual disease.

Significant number of complications occurred due to surgery combined with radiotherapy (70.58\%). Complications due to radiotherapy were mostly mild and included radiation mucositis or skin pigmentation. Chemotherapy was the most well tolerated treatment with only minor complications like alopecia and neutropenia which were managed successfully. Out of the 16 patients who received chemotherapy alone, only five patients developed complications. Complications of surgery included postmaxillectomy trismus (five cases) and ectropion of the lower lid due to scar contraction (six cases). One patient in whom maxillectomy with orbital exenteration was done, developed postoperative meningitis but recovered from it.

During the 2.5 years of our study period we lost five patients. A case of malignant schwannoma of the maxilla died after 1 year of completion of treatment due to distant metastasis, two cases of adenoid cystic carcinoma of the maxilla died at 1.5 and 2.5 years with liver metastasis, one case of esthesioneuroblastoma developed intracerebral metastasis and a patient of Hodgkins lymphoma died due to unrelated medical illness while still on chemotherapy.

\section{DISCUSSION}

Malignant tumors arising in the head and neck region account for $5.6 \%$ of all body tumors and less than $10 \%$ of them are nonsquamous in histology. ${ }^{1}$ According to Jacobs and Negandak ${ }^{2}$ the most common nonepithelial malignancy in the head and neck region are lymphomas. However, no study has been conducted on nonsquamous cell malignancies of the head and neck as a whole.
We found that the nose and paranasal sinus system was the most common sites of involvement, for the nonsquamous variety of tumors, in the head and neck region. In our study out of the 30 cases of tumors in the nose and paranasal sinuses, $22(46 \%)$ were in the maxillary antrum, five were in the nasal cavity $(10.42 \%)$, two in the ethmoid sinus (4.17\%) and one over the lateral wall of the nose (2.08\%).

Few studies have been conducted on the study of nonsquamous cell malignancies in the nose and paranasal sinus system or only pertaining to the maxillary sinus. Spiro et $\mathrm{al}^{6}$ found that $50 \%$ cases had tumors in the nasal cavity, $39 \%$ in the antrum, $9 \%$ in the ethmoid and $2 \%$ in the frontal sinus. On the other hand Fu and Perzin ${ }^{5}$ found that, of the 11 cases, seven involved the maxillary sinus, two involved the lateral wall of the nasal cavity, one involved the ethmoid sinus and one case involved multiple sinuses, the nasal cavity, skull base and the orbit.

In our study, adenoid cystic carcinoma was the commonest histopathological type found in the maxillary sinuses (41\%) followed by adenocarcinoma (23\%). Bhattacharya ${ }^{4}$ also found adenoid cystic carcinoma to be the commonest (34\%). However, Qureshi ${ }^{3}$ and $\mathrm{Kraus}^{7}$ found sarcomas to be the most common.

It is usually agreed upon, that the treatment for maxillary sinus tumors is surgical, except for some specific indications (e.g. lymphomas). Combination of surgery with radiotherapy or chemotherapy is advocated as and when appropriate. Surgery along with radiotherapy was the most commonly employed treatment in our study which is in keeping with Qureshi's study. ${ }^{3}$ However, they have not noted a single case having received only chemotherapy or only radiotherapy. This is because we have documented two cases of lymphoma, which underwent chemotherapy.

We documented a total of 12 cases of lymphomas of which seven were non-Hodgkins' lymphomas and five were Hodgkins' lymphomas. According to most literature, Hodgkins' lymphomas present in the supraclavicular or lower cervical lymph nodal groups as painless neck swellings. ${ }^{2}$ All our cases of Hodgkins' disease also presented with painless lymph nodal enlargement in the lower cervical group of nodes. Extranodal presentation of disease is seen in non-Hodgkins' lymphomas and infact all our cases were of extranodal presentation. Waldeyer's ring was the most common site in our study followed by the paranasal sinuses. These findings were consistent with the findings of other authors. $^{8-10}$

The survival statistics and recurrence rate of our study cannot be compared with other studies as the duration of our study has been 2.5 years and therefore it is not possible to calculate 5-year survival rates as of now. 


\section{CONCLUSION}

Nonsquamous cell cancers of the head and neck region are relatively uncommon tumors and have not been studied as exhaustively as their squamous cell counterparts. Most of the tumors are located in the nose and paranasal sinus region, maxilla being the most commonly affected. Lymphomas are the most common nonepithelial malignancies in the head and neck region. In the nose and paranasal sinuses, minor salivary gland tumors are the commonest nonsquamous cell cancers and among them adenoid cystic carcinomas are the most common.

Treatment modality has to be tailor made to the histopathological subtype of the tumor. However, surgery in combination with radiotherapy remains the most commonly employed treatment followed by chemotherapy. Response to treatment is quite good with maximum patients showing complete resolution of tumor at treatment completion. Mortality rate is significant, especially in the rare, aggressive type of pathologies and with locally advanced stage of the tumors.

Hence, there is a need for comprehensive studies which will help the clinician in suspecting these rare varieties of tumors and having statistically backed knowledge which will help in patient counseling and treatment.

\section{REFERENCES}

1. Montravadi RVP. Radiation therapy for nonsquamous cell tumors of the head and neck. Otolaryngol Clin North Am 1986 Nov;19(4):741-50.

2. Jacobs JR, Negendank WG. Lymphomas of the head and neck. In: Paparella, et al (Eds). Otolaryngology (3rd ed). WB Saunders 1991;3:2591-98.

3. Qureshi SS, Chaukar DA, Talole SD, Dcruz AK. Clinical characteristics and outcome of nonsquamous cell malignancies of the maxillary sinus. J Surg Oncol 2006;93:362-67.

4. Bhattacharya N. Survival and staging characteristics of the nonsquamous cell malignancies of the nose and paranosal sinuses. Arch Otolaryngol Head Neck Surg 2003;129:334-37.
5. Fu YS, Perzin KH. Nonepithelial tumors of the nasal cavity, paranasal sinuses and nasopharynx: A clinicopathological study. Cancer 1977;40:1314-17.

6. Spiro J, Spiro R, Soo KC, et al. Nonsquamous cell malignancies of the nose and paranasal sinuses. J Head Neck Surg 2006 Jul;17(2):114-18.

7. Kraus DH, Roberts JK, Medendorp SV, et al. Nonsquamous cell malignancies of the paranasal sinuses. Annals Otol Rhinol Laryngol 1990;99:5-11.

8. Cobleigh MA, Kennedy JL. Non-Hodgkins' lymphomas of the upper aerodigestive tract and salivary glands. Otolaryngol Clin North Am 1986 Nov;19(4):685-704.

9. Jacobs C, Hoppe RT. Non-Hodgkin's lymphomas of the head and neck extranodal sites. Int J Rad Oncol Biol Phys 1985 Feb;11(2):357-64.

10. Lee YY, Tassel PV, North LB, et al. Lymphomas of the head and neck: CT findings at initial presentation. AJR Am J Roentgenol 1987 Sep;149(3):575-81.

\section{ABOUT THE AUTHORS}

\section{Vipin Ram Ekhar (Corresponding Author)}

Assistant Professor, Department of Otolaryngology, Government Medical College, Nagpur, Maharashtra, India, e-mail: vipinekhar@yahoo.com

\section{Devendra M Mahore}

Associate Professor, Department of Otolaryngology, Government Medical College, Nagpur, Maharashtra, India

\section{Gauri M Kapre}

Senior Resident, Department of Otolaryngology, Government Medical College, Maharashtra, India

\section{Bhagyashree D Bokare}

Associate Professor, Department of Otolaryngology, Government Medical College, Nagpur, Maharashtra, India

\section{Surendra H Gawarle}

Professor and Head, Department of Otolaryngology, Government Medical College, Nagpur, Maharashtra, India 